\title{
Theoretical models for the selectivity of organic singlet and triplet photoreactions
}

\author{
Martin Klessinger \\ Organisch-Chemisches Institut der Westfal. Wilhelms-Universität \\ Corrensstr. 40, D-48149 Minster, Germany
}

\begin{abstract}
In nonadiabatic photoreactions in solution, the geometry at the decay region from the excited to the ground state is decisive for the outcome of the reaction. From a mechanistic point of view, this "reactive structure", i.e., the structure of the conical intersection accessible on the excited state of a singlet photoreaction or the region of geometries favorable for intersystem crossing in a triplet photoreaction, plays a similar role as the transition state in a thermal reaction. Consequences for the selectivity of singlet and triplet photoreactions are discussed.
\end{abstract}

\section{INTRODUCTION}

In a nonadiabatic photoreaction, four distinct steps may be distinguished (Fig. 1): 1) excitation from the ground state $S_{0}$ into the lowest singlet $\left(S_{1}\right)$ or triplet state $\left.\left(T_{1}\right), 2\right)$ movement (possibly over barriers) on the excited state potential energy surface (PES), 3 ) return to the $S_{0}$ state, and 4) relaxation on the ground state PES (ref. 1). In solution reactions, thermal equilibration is fast and the geometry of return from the excited

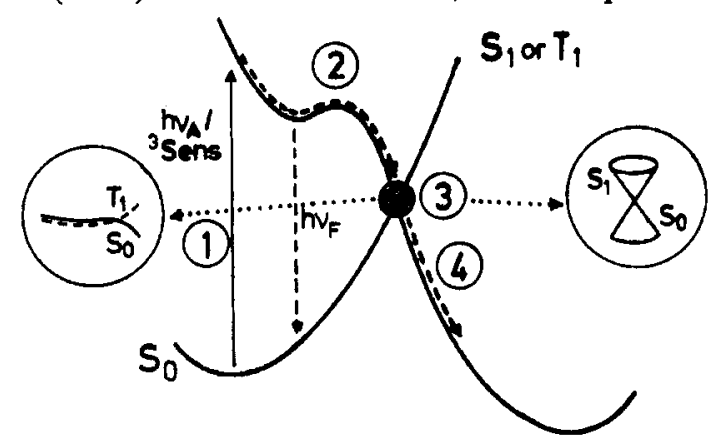

Fig. 1 The mechanism of nonadiabatic singiet and triplet photoreactions; decay back to the singlet state via a conical intersection or via ISC, respectively. to the ground state is of paramount importance for the outcome of the reaction. For a singlet reaction, this is a conical intersection (ref. 2), which from a mechanistic point of view plays a similar role as the transition state in a thermal reaction (ref. 3 ). In triplet photoreactions, the geometries most favorable for intersystem crossing (ISC) may be considered to be of similar relevance (ref. 4). Thus, the knowledge of the "reactive structure", i.e. the transition structure of a thermal reaction, the structure of the conical intersection accessible on the excited state PES of a singlet photoreaction or the geometries favorable for ISC in a triplet photoreaction, is decisive for the discussion and prediction of the selectivity of the reaction.

\section{SINGLET PHOTOREACTIONS}

Return from $S_{1}$ to $S_{0}$ occurs by passage through a funnel in the $S_{1}$ PES. Until recently, it was believed that the funnels mostly correspond to avoided surface crossings. A vibrational analysis at the "pericyclic minimum" e.g. of the [2+2]photoaddition of two ethylene molecules shows this stationary point to be a transition state rather then a minimum. The imaginary frequency corresponds to a rhomboidal distortion that leads to two equivalent true conical intersections (ref. 5). In fact, in a remarkable series of recent papers, Bernardi, Olivucci, Robb and their collaborators demonstrated that conical intersections are a common feature in most nonadiabatic singlet photoreactions (cf. ref. 2). The decay via a conical intersection is very fast and has been demonstrated to take place within a single vibrational period (ref. 6). Thus, the geometry at the conical intersection is decisive for product formation in the ground state.

The nature of the PES near a conical intersection has been discussed by Ruedenberg et al. (ref. 7). A conical intersection of two PES spans the $f-2$ dimensional intersection space $(f=3 N-6)$. The remaining two linearly independent coordinates $x_{1}$ and $x_{2}$ define the branching space, with $x_{1}$ and $x_{2}$ being the gradient difference vector and the nonadibatic coupling vector, respectively. When the energy of the two states is plotted against $x_{1}$ and $x_{2}$, the corresponding PES have the shape of a double cone. 
Several models have been proposed to predict the structure of the conical intersection. Robb and Bernardi have shown that in the framework of a $2 \times 2$ VB model the nature of the surface-crossing region may be predicted on the basis of the geometry dependence of the VB exchange integrals (ref. 8). Particularly useful is the two-electron two-orbital model (2-in-2 model) of Michl and Bonačić-Koutecký (ref. 9), which is applicable both to singlet and triplet photoreactions. Two electrons in two orbitals yield three singlet and one triplet configuration. Using the most localized orthogonal orbitals, the Hamiltonian matrix is diagonal for a perfect biradical and the three singlet functions describe eigenstates. In a homosymmetric biradicaloid, a covalent perturbation $\gamma$ (equivalent to twice the Hückel resonance integral between the localized orbitals) mixes the covalent and one of the hole-pair state functions of the perfect biradical, stabilizing its ground state $S_{0}$ and destabilizing its $S_{2}$ relative to the triplet state. In a heterosymmetric biradicaloid, a polarizing perturbation $\delta$ (equivalent to the difference $\delta \alpha$ between the Hückel Coulomb integrals of the localized orbitals) mixes the two hole-pair state functions of the perfect biradical, resulting in an increase and a decrease in the energies of $S_{2}$ and $S_{1}$, respectively. When the perturbation becomes equal to a critical value $\delta_{0}$, it causes a surface crossing between $S_{1}$ and $S_{0}$.

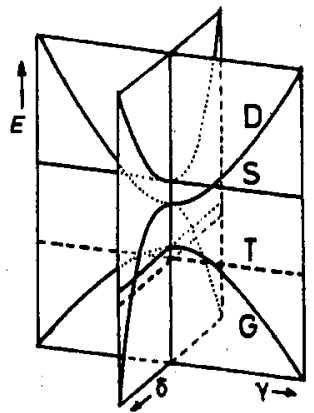

Fig. 2 The 2-in-2 model for a conical intersection. Starting from the pericyclic geometry a covalent interaction $\gamma$ mixes the $G$ and $D$ states, producing the Oosterhoff diagram, while the polarizing perturbation $\delta$ mixes the $S$ and $D$ states leading to a conical intersection at the "critically heterosymmetric" geometry $\left(\delta=\delta_{0}\right)$.

The general case with $\gamma \neq 0$ and $\delta \neq 0$ is shown in Fig. 2 . The $\gamma, E$ plane shows the well-known Oosterhoff diagram (ref. 10). Going from the pericyclic geometry along the $\delta$ coordinate, a surface crossing is reached, depicting a $S_{1}-S_{0}$ conical intersection corresponding to a critically heterosymmetric biradicaloid: $\mathbf{x}_{1}$ is the $\delta$ coordinate (i.e., the direction of the fastest change in the energy difference between the nonbonding orbitals) and $x_{2}$ the $\gamma$ coordinate (i.e., the direction of the fastest change in the degree of interaction between these two orbitals).

A simple example is provided by the [2+2]photoaddition of two ethylene molecules (ref. 11). Plotting the energy against the distance between the two molecules at rectangular geometries (covalent interaction $\gamma$ ) yields the usual state diagram for pericyclic photoreactions. Using as a second coordinate a rhomboidal distortion, which introduces a diagonal interaction and therefore differentiates the otherwise degenerate energies of the two localized orbitals (polarizing perturbation $\delta$ ), two conical intersections equivalent by symmetry result that correspond either to an enhanced 1,3 or an enhanced 2,4 interaction.

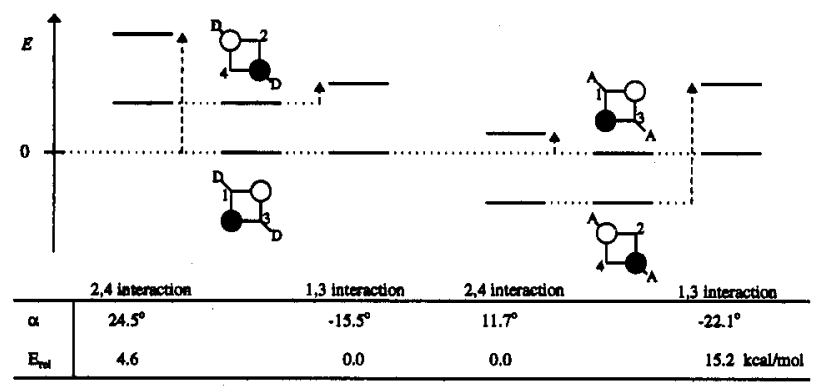

Fig. 3 Syn head-to-tail [2+2] cycloaddition of two donor (D) or acceptor (A) substituted ethylenes. Schematic representation of the energies of the cyclobutadiene-like nonbonding biradicaloid orbitals. The effect of substituents D and A on the orbital energies is incorporated in the levels shown in the center of each half of the diagram. The effect of diagonal interaction required to reach the conical intersection at the critical value $\delta_{0}$ is indicated by broken arrows. Computational results for $\mathrm{D}=\mathrm{NH}_{2}$ and $\mathrm{A}=\mathrm{CN}$ are given below, positive values of $\alpha$ correspond to a rhomboidal distortion which brings the substituted atoms closer to each other.

In the case of the [2+2]photoaddition of substituted ethylenes (ref. 5), these two situations are no longer equivalent for head-to-tail addition (1,3 arrangement of the substituents), and the diagonal interaction may either reinforce or counteract the effect of the substituents, depending on which of the two diagonals has been shortened. Two conical intersections will result, one at a less and one at a more diagonally distorted 
geometry than for the unsubstituted ethylene. This is confirmed by the results of calculations shown in Fig. 3 (ref. 12). For small rhomboidal distortions, the peripheral bonding will dominate at the geometry after return to $S_{0}$, and the formation of the head-to-tail product is likely to be favored. The diagonal bonding will dominate for large distortions, however, and head-to-head product could possibly be formed by $x[2+2]$ cycloaddition, i.e. by formation of the diagonal bond and subsequent closure of the other. Thus, the result of the photoaddition will depend on extent of rhomboidal distortion, i.e. on the structure of the conical intersection.

However, the structure of the conical intersection is not the only factor that determines the selectivity of a singlet photoreaction. From the definition of the branching space it is evident that a conical intersection provides two linearly independent reaction-path directions $x_{1}$ and $x_{2}$. In contrast to a thermal reaction, where the initial motion after passage through a transition state is characterized by a single vector $\mathbf{q}_{R}$ (the normal coordinate of the imaginary vibrational frequency), the photochemical reaction path in the immediate vicinity of the apex of the cone can follow any direction in the $x_{1}, x_{2}$ plane and may reach different reaction valleys that develop on the $S_{0}$ surface and lead to different products.

This is best illustrated by considering the cis-trans isomerization of ethylene: For $90^{\circ}$-twisted ethylene the $\mathrm{p}$

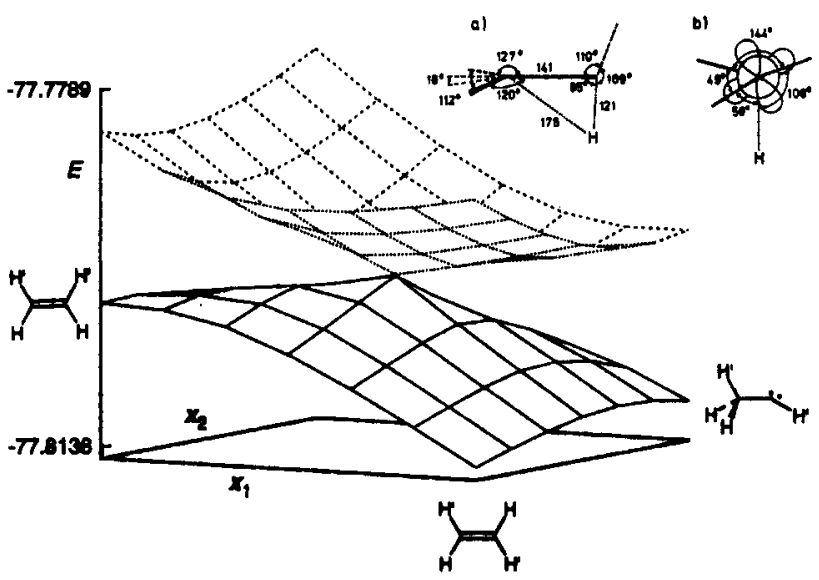

Fig. 4 CASSCF results for the geometry (a) side view and b) Newman projection] and the PES of the $S_{0}-S_{1}$ conical intersection of ethylene; groundstate pathways may lead to cis-trans isomerization, $[1,2]$ hydrogen shift to methyl carbene or back to the reactant.

se to the come tip either the cis trans isomerization product, or mo on a barrierless ground-state relaxation pathway (ref. 14).

\section{TRIPLET PHOTOREACTIONS}

In triplet photoreactions, the knowledge of the geometries at which ISC allows for a return from $T_{1}$ to $S_{0}$ is important for an understanding of the nature of the products. There are three mechanisms of ISC: solventinduced spin relaxation by independent spin-flips, the hyperfine coupling that is important in biradicals in which the two radical centers are relatively far apart, and SOC that is dominant in short biradicals, as is observed in intermediates in numerous photochemical reactions (ref. 1). In the following we will concentrate on the SOC mechanism only.

Two conditions have to be satisfied for the SOC mechanism to become efficient: the $T_{1}-S_{0}$ energy gap has to be small and SOC should be large (ref. 15). Thus, to be able to estimate the overall ISC probability, one has to determine the PES to locate the accessible areas of small $T_{1}-S_{0}$ separation as well as to evaluate the SOC for each geometry of interest. Recently, we presented a formalism for the determination of SOC in the comtext of configuration interaction calculations based on Rumer spin eigenfunctions and the second quantization approach (ref. 16). This formalism has been implemented for the semiempirical MNDOC-CI method (ref. 17) and allows for routine calculations of PES and SOC surfaces at the same level of theory. The method has been used to study the magnitude and geometry dependence of SOC in carbene, ethylene and trimethylene (ref. 16). The results are in good accord with available ab initio data, yielding e.g. for twisted ethylene a maximum value $S O C=0.44 \mathrm{~cm}^{-1}$ as compared to the MCSCF value $S O C=0.6 \mathrm{~cm}^{-1}$ (ref. 18 ). 
SOC in 1,n-biradicals may also be discussed on the basis of the two-electron two-orbital model (ref. 1); in a recent detailed analysis the model was shown to be very successful in predicting and rationalizing the structural dependence of SOC in biradicals (ref. 19).

In order to study the ring-closure reaction of trimethylene, PES as well as the SOC surfaces were calculated for the variation of the CCC angle $\gamma$ and the conrotatory $(\alpha=\beta)$ and disrotatory $\left(\beta=180^{\circ}-\alpha\right)$ motion of the terminal methylene groups (ref. 16). Since both motions yield very similar results, the $T_{1}$ and SOC surfaces are shown in Fig. 5 for the conrotatory mode only. The minimum energy valley of the $T_{1}$ PES that corresponds to nearly free rotation of the radical centers is unfavorable for ISC since the $S_{0}$ state lies energetically above the $T_{1}$ state. However, in the region of large $S O C$, where the terminal methylene groups are rotated by $\alpha>45^{\circ}$ toward an face-to-face arrangement, decreasing the $\mathrm{CCC}$ angle to a value $\gamma \approx 105^{\circ}$ rises the energy only slightly $(1-2 \mathrm{kcal} / \mathrm{mol})$ and leads to $\mathrm{T}_{1}-\mathrm{S}_{0}$ degeneracy $\left(E_{\mathrm{ST}}=0\right)$ and therefore to geometries very favorable to ISC.
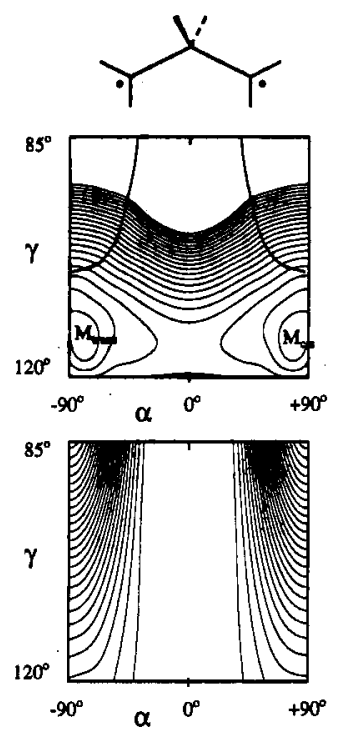
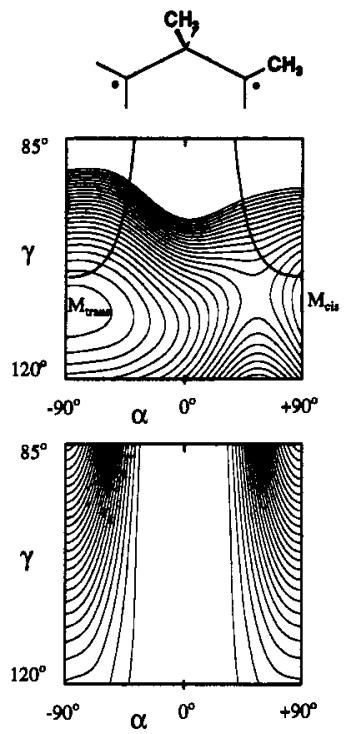

Fig. 5 Triplet PE and SOC surfaces for the ring-closure reaction of trimethylene (left) and 1,2-dimethyltrimethylene, as a function of the CCC valence angle $\gamma$ and the conrotatory motion $(\alpha=\beta)$ of the radical centers. SOC values vary between $0 \mathrm{~cm}^{-1}$ in the middle of the diagram and $7.5 \mathrm{~cm}^{-1}$ at the upper right and left corners. $\mathrm{T}_{1}-\mathrm{S}_{0}$ intersection $E_{\mathrm{TS}}=0$ is indicated by heavy lines.

In 1,2-dimethyltrimethylene there are two different modes of conrotatory motion of the radical centers, leading to stereoisomeric cyclization products: rotation by positive $(\alpha>0)$ and negative $(\alpha$ $<0)$ values of the rotational angle yields cis- and trans-dimethylcyclopropane, respectively. $\mathrm{T}_{1}$ and SOC surfaces are therefore shown in Figure 5 for the range $\alpha=-90^{\circ}$ to $\alpha=90^{\circ}$ (ref. 20). From a comparison of the results for the unsubstituted and the substituted trimethylene it is evident that to a first approximation the methyl substituents do not at all affect the $S O C$ values, while steric effects appreciably change the appearance of the valley on the $\mathrm{T}_{1}$ PES. For the syn mode of rotation a barrier of approximately $3.4 \mathrm{kcal} / \mathrm{mol}$ is found to separate the local minimum $M_{\text {cis }}$ at $\alpha=90^{\circ}$ from the planar structure $\left(\alpha=0^{\circ}\right)$ that is $1.0 \mathrm{kcal} / \mathrm{mol}$ lower in energy. For the anti mode of rotation, however, the valley descends practically without a barrier toward the minimum $\mathrm{M}_{\text {trans }}$ at $\alpha=-90^{\circ}$, $5.0 \mathrm{kcal} / \mathrm{mol}$ lower in energy than $\mathrm{M}_{c i s}$. By decreasing the bond angle $\gamma$, geometries are accessible both from $M_{c i s}$ and $M_{\text {trans }}$ at which $S_{0}$ and $T_{1}$ are degenerate $\left(E_{\mathrm{ST}}=0\right)$ and $S O C$ is appreciable $\left(S O C>2 \mathrm{~cm}^{-1}\right.$ ), the necessary energies being smaller than $1 \mathrm{kcal} / \mathrm{mol}$. For rotational angles $|\alpha|$ $<45^{\circ}$, however, much larger energies $\left(>10 \mathrm{kcal} / \mathrm{mol}\right.$ ) are required and geometries with $S O C<1 \mathrm{~cm}^{-1}$ are reached.

From these results, it can be concluded that, as in the unsubstituted trimethylene, the reactive structure of optimal ISC is characterized by a face-to-face orientation of the radical centers and a CCC angle $\gamma$ slightly smaller than for the triplet minima. As the singlet PES drops steeply for small values of $\gamma$, the triplet state yields preferably cyclic products. The conditions for optimal ISC are similar for both minima $\mathbf{M}_{c i s}$ and $\mathrm{M}_{\text {trans }}$; therefore, the stereochemical differentiation between cis- and trans-substituted products is due, in this case, to the energy difference of the two minima $\mathbf{M}_{c i s}$ and $\mathbf{M}_{\text {trans }}$. This explains the experimental observation that the triplet sensitized photoreaction of $c i s-$ as well as trans-3,4-dimethyl-1-pyrazoline yields preferably trans-1,2-dimethylcyclopropane, and negligible amounts of acyclic products, in contrast to the singlet photoreaction, which occurs preferably with retention of the configuration and yields appreciable amounts of acyclic products (ref. 21).

For tetramethylene SOC and $T_{1}$ surfaces were calculated as a function of the rotational angels $\alpha$ and $\bar{\beta}$ of the terminal $\mathrm{CH}_{2}$ groups for different values of the dihedral angle $\theta$ as shown in Fig. 6 . They demonstrate that the behavior of a triplet 1,4-biradical is determined by two opposing trends: SOC is large at syn geometries, 
a)
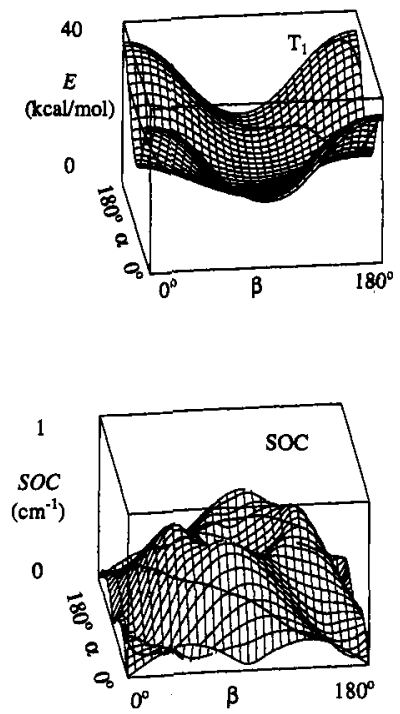

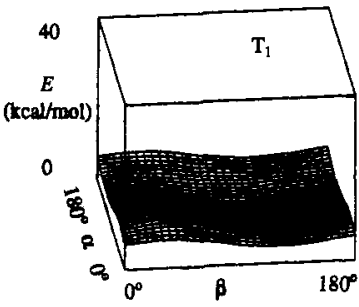

b)

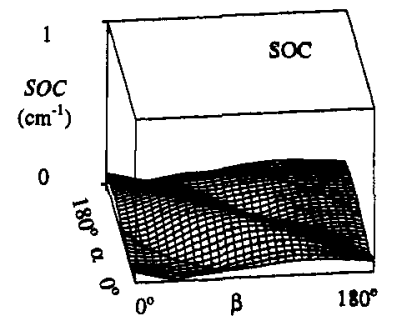

c)
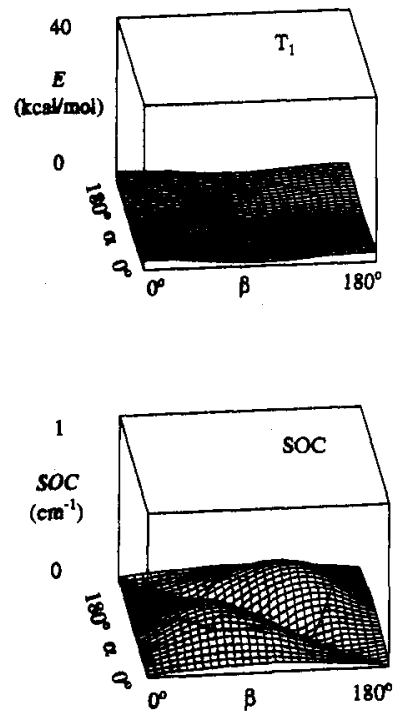

Fig. 6 Triplet PE and SOC surfaces for tetramethylene for rotations $\alpha$ and $\beta$ of the radical centers; a) syn conformation (CCCC dihedral angle $\left.\theta=0^{\circ}\right)$, b) gauche conformation $\left(\theta=60^{\circ}\right)$ and c) anti conformation $\left(\theta=180^{\circ}\right)$.

but regions of $\mathrm{T}_{1}-\mathrm{S}_{0}$ degeneracy are easiest to reach at anti geometries. These results account for the experimental results (ref. 23): Due to the shape of the $\mathrm{T}_{1}$ surface the system will reach the region of anti geometries very quickly. Here, $S O C$ values are only about one tenth of the maximum value, but the high probability of finding the system at these geometries together with the small $E_{S T}$ for nearly all values of the roational angles $\alpha$ and $\beta$ will favor the ISC process, yielding mainly open-chain products. Cyclic products, on the other hand, can result only from ISC in the region of syn geometries. The low stereospecifity of the triplet ring-closure reaction is then due to the fact that geometries with $E_{\mathrm{ST}} \approx 0$ and large SOC values are found in this region to occur at face-to-edge conformations, i.e., at geometries at which just one of the terminal methylene groups has been rotated by approximately $90^{\circ}$.<smiles>O=CC1CCCCC1Cc1ccccc1</smiles>

1

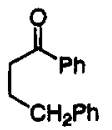

2

The results also provide a rationalization of the rather surprising finding of Caldwell et al. (ref. 24), that the ISC rate constant for the Norrish type II reaction of 1 , which is conformationally fixed at $\theta=60^{\circ}$, and of the flexible analogue 2 are nearly the same. In 1 , rotation around one of the terminal $C C$ bonds will lead to $T_{1}-S_{0}$ degeneracy with $S O C=0.28 \mathrm{~cm}^{-1}$, which corresponds exactly to the situation in 2, which will prefer the anti conformation where near $S_{0}-T_{1}$ degeneracies are found for any $\alpha$ and $\beta$ with $S O C<0.3 \mathrm{~cm}^{-1}$. This explains why other effects like the solvents affect the ISC rate to a larger extent than fixation of the biradical to $\theta=60^{\circ}$.

\section{CONCLUSIONS}

The knowledge of the "reactive structure", i.e. the transition structure of a thermal reaction, the structure of the conical intersection accessible on the excited state PES of a singlet photoreaction or the geometries favorable for ISC in a triplet photoreaction, is decisive for the discussion and prediction of the stereochemical outcome of the reaction.

For a thermal reaction, the system will be moving very slowly along the negative curvature at the transition state and following the minimum energy path up to products. At a conical intersection the situation is quite different. Trajectories passing exactly through the tip of the cone proceed undisturbed and follow the typically quite steep slope of the cone wall, thus converting electronic energy into energy of nuclear motion, which will be superimposed on the motion in the $\mathbf{x}_{1}, \mathbf{x}_{2}$ plane generated by the passage through the conical intersection. The actual prediction of relative or absolute product quantum yields for a singlet photoreaction requires a dynamic description of both the adiabatic motion on the excited and ground state PES and the nonadiabatic process describing the radiationless decay from the upper to the lower state. The efficiency of the 
various processes will thus depend on both static and dynamic factors. The structure of the conical intersection point where the ground-state pathways originate is an important static factor, that is easily predicted by quantum chemical calculations, while a quantitative treatment of the dynamics of the reaction under investigation is still a difficult task for organic systems.

In triplet photoreactions, the decay from the $T_{1}$ to the $S_{0}$ surface occurs at geometries favorable for ISC. In general, these geometries do not coincide with the minima on the $T_{1}$ surface. Thus motions over barriers are required in order to reach these regions, and the decay structure will depend on the accessibility and on the probability of finding the system at these geometries, at which the $S_{0}$ surface will typically slope steeply toward a product minimum, with no barrier in the way. Thus, ISC in triplet biradicals normally produces closed-shell singlet products and not floppy singlet biradicals (ref. 25).

In summary, the selectivity of organic singlet photoreactions is determined by the location of the conical intersection(s) as well as the dynamics on the excited and ground state PES, while in organic triplet photoreactions product formation depends typically only on the accessible regions favorable for ISC, that is on the energetics and dynamics on the $\mathrm{T}_{1}$ PES.

\section{REFERENCES}

1. M. Klessinger and J. Michl. Excited States and Photochemistry of Organic Molecules. VCH Publishers, New York (1995).

2. M. Klessinger. Angew. Chem. Int. Ed. Engl. 34, 549 (1995), and ref. therein.

3. M. Olivucci, F. Bernardi, S. Ottani and M. A. Robb. J. Am. Chem. Soc. 116, 2034 (1994).

4. M. Klessinger, M. Böckmann and J. Mählmann. J. Inf. Rec. Mats. 21, 549 (1994).

5. J. Bentzien and M. Klessinger. J. Org. Chem. 59, 4887 (1994).

6. U. Manthe and H. Köppel. J. Chem. Phys. 93,1669 (1990).

7. G. J. Atchity, S. S. Xantheas and K. Ruedenberg. J. Chem. Phys. 95,1862 (1991).

8. F. Bernardi, M. Olivucci and M. A. Robb, Israel J. Chemistry 33, 265 (1993).

9. a) V. Bonacić-Koutecký, J. Koutecký and J. Michl. Angew. Chem. Int. Ed. Engl. 26, 170 (1987); b) J. Michl and V. BonacicKoutecký. Electronic Aspects of Organic Photochemistry. Wiley, New York (1990).

10. W. T. A. M. van der Lugt and L. J. Oosterhoff. J. Am. Chem. Soc. 91,6042 (1969).

11. F. Bernardi, S. De, M. Olivucci and M. A. Robb. J. Am. Chem. Soc. 112, 1737 (1990).

12. C. Hoinka and M. Klessinger, to be published.

13. 1. Otmine. J. Chem. Phys. 83, 2348 (1985); see also ref. $9 \mathrm{~b}$.

14. L. Freund and M. Klessinger, to be published.

15. S. P. McGlynn, T. Azumi and M. Kinoshita. Molecular Spectroscopy of the Triplet State. Prentice-Hall, Englewood Cliffs (1969).

16. M. Böckmann, M. Klessinger and M. C. Zerner. J. Phys. Chem. (in press).

17. M. Klessinger, T. Pötter and C. van Wüllen. Theor. Chim. Acta 80, 1 (1991).

18. R. A. Caldwell, L. Carlacci, C. E. Doubleday Jr., I. R. Furlani, H. F. King and J. W. McIver Jr. J. Am. Chem. Soc. 110, 6901 (1988).

19. J. Michl. J. Am. Chem. Soc. 118, 3568 (1969).

20. M. Böckmann and M. Klessinger, submitted.

21. R. Moore, A. Mishra and R. I. Crawford. Canad. J. Chem. 46, 3305 (1968).

22. M. Böckmann, J. Mählmann and M. Klessinger, to be published.

23. a) P. D. Bartlett and N. A. Porter, J. Am. Chem. Soc. 90, 5317 (1968); b) P. G. Schultz and P.B. Dervan. J. Am. Chem. Soc. 104, 6660 (1982).

24. R. A. Caldwell, N. S. Dhawan and T. Majma. J. Am. Chem. Soc. 106, 6454 (1984).

25. P. J. Wagner. Acc. Chem. Res. 22, 83 (1989). 